Research Article

\title{
Connectedness and Compactness of Weak Efficient Solutions for Set-Valued Vector Equilibrium Problems
}

\author{
Bin Chen, Xun-Hua Gong, and Shu-Min Yuan \\ Department of Mathematics, Nanchang University, Nanchang 330047, China \\ Correspondence should be addressed to Xun-Hua Gong, xunhuagong@gmail.com
}

Received 1 November 2007; Revised 17 July 2008; Accepted 5 September 2008

Recommended by C. E. Chidume

We study the set-valued vector equilibrium problems and the set-valued vector HartmanStampacchia variational inequalities. We prove the existence of solutions of the two problems. In addition, we prove the connectedness and the compactness of solutions of the two problems in normed linear space.

Copyright (C) 2008 Bin Chen et al. This is an open access article distributed under the Creative Commons Attribution License, which permits unrestricted use, distribution, and reproduction in any medium, provided the original work is properly cited.

\section{Introduction}

We know that one of the important problems of vector variational inequalities and vector equilibrium problems is to study the topological properties of the set of solutions. Among its topological properties, the connectedness and the compactness are of interest. Recently, Lee et al. [1] and Cheng [2] have studied the connectedness of weak efficient solutions set for single-valued vector variational inequalities in finite dimensional Euclidean space. Gong [3-5] has studied the connectedness of the various solutions set for single-valued vector equilibrium problem in infinite dimension space. The set-valued vector equilibrium problem was introduced by Ansari et al. [6]. Since then, Ansari and Yao [7], Konnov and Yao [8], Fu [9], Hou et al. [10], Tan [11], Peng et al. [12], Ansari and Flores-Bazán [13], Lin et al. [14] and Long et al. [15] have studied the existence of solutions for set-valued vector equilibrium and set-valued vector variational inequalities problems. However, the connectedness and the compactness of the set of solutions to the set-valued vector equilibrium problem remained unstudied. In this paper, we study the existence, connectedness, and the compactness of the weak efficient solutions set for set-valued vector equilibrium problems and the set-valued vector Hartman-Stampacchia variational inequalities in normed linear space. 


\section{Preliminaries}

Throughout this paper, let $X, Y$ be two normed linear spaces, let $A$ be a nonempty subset of $X$, let $F: A \times A \rightarrow 2^{Y}$ be a set-valued map, and let $C$ be a closed convex pointed cone in $Y$. such that

We consider the following set-valued vector equilibrium problem (SVEP): find $\bar{x} \in A$,

$$
F(\bar{x}, y) \cap(-\operatorname{int} C)=\varnothing \quad \forall y \in A
$$

Definition 2.1. Let int $C \neq \varnothing$. A vector $x \in A$ satisfying

$$
F(x, y) \cap(-\operatorname{int} C)=\varnothing \quad \forall y \in A
$$

is called a weak efficient solution to the SVEP. Denote by $V_{w}(A, F)$ the set of all weak efficient solutions to the SVEP.

Let $Y^{*}$ be the topological dual space of $Y$. Let

$$
C^{*}=\left\{f \in Y^{*}: f(y) \geq 0 \forall y \in C\right\}
$$

be the dual cone of $C$.

Definition 2.2. Let $f \in C^{*} \backslash\{0\}$. A vector $x \in A$ is called an $f$-efficient solution to the SVEP if

$$
f(F(x, y)) \geq 0 \quad \forall y \in A,
$$

where $f(F(x, y)) \geq 0$ means that $f(z) \geq 0$, for all $z \in F(x, y)$. Denote by $V_{f}(A, F)$ the set of all $f$-efficient solutions to the SVEP.

Definition 2.3. Let $A$ be a nonempty convex subset in $X$. A set-valued map $F: A \times A \rightarrow 2^{Y}$ is called to be $C$-convex in its second variable if, for each fixed $x \in A$, for every $y_{1}, y_{2} \in A$, $t \in[0,1]$, the following property holds:

$$
t F\left(x, y_{1}\right)+(1-t) F\left(x, y_{2}\right) \subset F\left(x, t y_{1}+(1-t) y_{2}\right)+C
$$

Definition 2.4. Let $A$ be a nonempty convex subset in $X$. A set-valued map $F: A \times A \rightarrow 2^{Y}$ is called to be $C$-concave in its first variable if, for each fixed $y \in A$, for every $x_{1}, x_{2} \in A$, $t \in[0,1]$, the following property holds:

$$
F\left(t x_{1}+(1-t) x_{2}, y\right) \subset t F\left(x_{1}, y\right)+(1-t) F\left(x_{2}, y\right)+C
$$

Definition 2.5. Let $A$ be a nonempty subset of $X$. Let $T: A \rightarrow 2^{L(X, Y)}$ be a set-valued map, where $L(X, Y)$ is the space of all bounded linear operators from $X$ into $Y$ (let $L(X, Y)$ be equipped with operator norm topology). Set $(T x, y)=\{(s, y): s \in T x\}, x, y \in A$. 
(i) Let $A$ be a convex subset of $X$. T is said to be $v$-hemicontinuous if, for every pair of points $x, y \in A$, the set-valued map

$$
J(\alpha):=(T(\alpha y+(1-\alpha) x), y-x), \quad \alpha \in[0,1]
$$

is lower semicontinuous at 0 .

(ii) Let $f \in C^{*} \backslash\{0\}$. T is said to be $f$-pseudomonotone on $A$ if, for every pair of points $x, y \in A, f((s, y-x)) \geq 0$, for all $s \in T x$, then $f\left(\left(s^{\prime}, y-x\right)\right) \geq 0$, for all $s^{\prime} \in T y$.

The definition of $v$-hemicontinuity was introduced by Lin et al. [14].

Definition 2.6. Let $X$ be a Hausdorff topological vector space and let $K \subset X$ be a nonempty set. $G: K \rightarrow 2^{X}$ is called to be a KKM map if for any finite set $\left\{x_{1}, \ldots, x_{n}\right\} \subset K$ the relation

$$
\operatorname{co}\left\{x_{1}, \ldots, x_{n}\right\} \subset \bigcup_{i=1}^{n} G\left(x_{i}\right)
$$

holds, where $\operatorname{co}\left\{x_{1}, \ldots, x_{n}\right\}$ denoted the convex hull of $\left\{x_{1}, \ldots, x_{n}\right\}$.

For the definition of the upper semicontinuity and lower semicontinuity, see [16].

The following FKKM theorem plays a crucial role in this paper.

Lemma 2.7. Let $X$ be a Hausdorff topological vector space. Let $K$ be a nonempty convex subset of $X$, and let $G: K \rightarrow 2^{K}$ be a KKM map. If for each $x \in K, G(x)$ is closed in $X$, and if there exists a point $x_{0} \in K$ such that $G\left(x_{0}\right)$ is compact, then $\bigcap_{x \in K} G(x) \neq \varnothing$.

By definition, we can get the following lemma.

Lemma 2.8. Let $A$ be a nonempty convex subset of $X$. Let $F: A \times A \rightarrow 2^{Y}$ be a set-valued map, and let $C \subset Y$ be a closed convex pointed cone. Moreover, suppose that $F(x, y)$ is $C$-convex in its second variable. Then, for each $x \in A, F(x, A)+C$ is convex.

\section{Scalarization}

In this section, we extend a result in [3] to set-valued map.

Theorem 3.1. Suppose that int $C \neq \varnothing$, and that $F(x, A)+C$ is a convex set for each $x \in A$. Then

$$
V_{w}(A, F)=\bigcup_{f \in C^{*} \backslash\{0\}} V_{f}(A, F)
$$

Proof. It is clear that

$$
V_{w}(A, F) \supset \bigcup_{f \in C^{*} \backslash\{0\}} V_{f}(A, F)
$$


Now we prove that

$$
V_{w}(A, F) \subset \bigcup_{f \in C^{*} \backslash\{0\}} V_{f}(A, F)
$$

Let $x \in V_{w}(A, F)$. By definition, $F(x, y) \cap(-\operatorname{int} C)=\varnothing$, for all $y \in A$. Thus

$$
F(x, A) \cap(-\operatorname{int} C)=\varnothing .
$$

As $C$ is a convex pointed cone, we have

$$
(F(x, A)+C) \cap(-\operatorname{int} C)=\varnothing .
$$

By assumption, $F(x, A)+C$ is a convex set. By the separation theorem of convex sets, there exist some $f \in Y^{*} \backslash\{0\}$, such that

$$
\inf \{f(F(x, y)+c): y \in A, c \in C\} \geq \sup \{f(-c): c \in C\}
$$

By (3.6), we obtain that $f \in C^{*} \backslash\{0\}$ and

$$
f(F(x, y)) \geq 0 \quad \forall y \in A .
$$

Therefore, $x \in V_{f}(A, F)$. Hence $V_{w}(A, F) \subset \bigcup_{f \in C^{*} \backslash\{0\}} V_{f}(A, F)$. Thus we have

$$
V_{w}(A, F)=\bigcup_{f \in C^{*} \backslash\{0\}} V_{f}(A, F)
$$

\section{Existence of the weak efficient solutions}

Theorem 4.1. Let $A$ be a nonempty closed convex subset of $X$ and let $C \subset Y$ be a closed convex pointed cone with int $C \neq \varnothing$. Let $F: A \times A \rightarrow 2^{Y}$ be a set-valued map with $F(x, x) \subset C$ for all $x \in A$. Suppose that for each $y \in A, F(\cdot, y)$ is lower semicontinuous on $A$, and that $F(x, y)$ is $C$-convex in its second variable. If there exists a nonempty compact subset $D$ of $A$, and $y^{\prime} \in D$, such that $F\left(x, y^{\prime}\right) \cap(-\operatorname{int} C) \neq \varnothing$, for all $x \in A \backslash D$, then, for any $f \in C^{*} \backslash\{0\}, V_{f}(A, F) \neq \varnothing, V_{f}(A, F) \subset D$, $V_{w}(A, F) \neq \varnothing$, and $V_{w}(A, F) \subset D$.

Proof. Let $f \in C^{*} \backslash\{0\}$. Define the set-valued map $G: A \rightarrow 2^{A}$ by

$$
G(y)=\{x \in A: f(F(x, y)) \geq 0\}, \quad y \in A \text {. }
$$

By assumption, $y \in G(y)$, for all $y \in A$, so $G(y) \neq \varnothing$. We claim that $G$ is a KKM map. Suppose to the contrary that there exists a finite subset $\left\{y_{1}, \ldots, y_{n}\right\}$ of $A$, and there exists 
$\bar{x} \in \operatorname{co}\left\{y_{1}, \ldots, y_{n}\right\}$ such that $\bar{x} \notin \bigcup_{i=1}^{n} G\left(y_{i}\right)$. Then $\bar{x}=\sum_{i=1}^{n} t_{i} y_{i}$ for some $t_{i} \geq 0,1 \leq i \leq n$, with $\sum_{i=1}^{n} t_{i}=1$, and $\bar{x} \notin G\left(y_{i}\right)$, for all $i=1, \ldots, n$. Then there exist $z_{i} \in F\left(\bar{x}, y_{i}\right)$, such that

$$
f\left(z_{i}\right)<0 \quad \forall i=1, \ldots, n
$$

As $F(x, y)$ is $C$-convex in its second invariable, we can get that

$$
t_{1} F\left(\bar{x}, y_{1}\right)+t_{2} F\left(\bar{x}, y_{2}\right)+\cdots+t_{n} F\left(\bar{x}, y_{n}\right) \subset F(\bar{x}, \bar{x})+C
$$

By (4.3), we know that there exist $z \in F(\bar{x}, \bar{x}), c \in C$, such that

$$
t_{1} z_{1}+t_{2} z_{2}+\cdots t_{n} z_{n}=z+c
$$

Hence $f(z+c)=f\left(t_{1} z_{1}+t_{2} z_{2}+\cdots t_{n} z_{n}\right)$. By assumption, we have $f(z+c) \geq 0$. By (4.2), however, we have $f\left(t_{1} z_{1}+t_{2} z_{2}+\cdots t_{n} z_{n}\right)<0$. This is a contradiction. Thus $G$ is a KKM map. Now we show that for each $y \in A, G(y)$ is closed. For any sequence, $\left\{x_{n}\right\} \subset G(y)$ and $x_{n} \rightarrow x_{0}$. Because $A$ is a closed set, we have $x_{0} \in A$. By assumption, for each $y \in A, F(\cdot, y)$ is lower semicontinuous on $A$, then by [16], for each fixed $y \in A$, and for each $z_{0} \in F\left(x_{0}, y\right)$, there exist $z_{n} \in F\left(x_{n}, y\right)$, such that $z_{n} \rightarrow z_{0}$. Because $\left\{x_{n}\right\} \subset G(y)$, we have

$$
f\left(F\left(x_{n}, y\right)\right) \geq 0 .
$$

Thus $f\left(z_{n}\right) \geq 0$. By the continuity of $f$ and $z_{n} \rightarrow z_{0}$, we have $f\left(z_{0}\right) \geq 0$. By the arbitrariness of $z_{0} \in F\left(x_{0}, y\right)$, we have $f\left(F\left(x_{0}, y\right)\right) \geq 0$, that is, $x_{0} \in G(y)$. Hence $G(y)$ is closed. By the assumption, we have $G\left(y^{\prime}\right) \subset D$, and $G\left(y^{\prime}\right)$ is closed. Since $D$ is compact, $G\left(y^{\prime}\right)$ is compact. By Lemma 2.7, we have $\bigcap_{y \in A} G(y) \neq \varnothing$. Thus there exists $x \in \bigcap_{y \in A} G(y)$. This means that

$$
f(F(x, y)) \geq 0 \quad \forall y \in A .
$$

Therefore, $x \in V_{f}(A, F)$. Next we show that $V_{f}(A, F) \subset D$. If $x \in V_{f}(A, F)$, then $x \in$ $\bigcap_{y \in A} G(y) \subset G\left(y^{\prime}\right) \subset D$. It follows from $V_{f}(A, F) \subset V_{w}(A, F)$ that $V_{w}(A, F) \neq \varnothing$, and by Theorem 3.1, we have $V_{w}(A, F) \subset D$.

Theorem 4.2. Let $A$ be a nonempty closed convex subset of $X$ and let $C \subset Y$ be a closed convex pointed cone with int $C \neq \varnothing$. Let $f \in C^{*} \backslash\{0\}$. Assume that $T: A \rightarrow 2^{L(X, Y)}$ is a v-hemicontinuous, $f$-pseudomonotone mapping. Moreover, assume that the set-valued map $F: A \times A \rightarrow 2^{\gamma}$ defined by $F(x, y)=(T x, y-x)$ is $C$-convex in its second variable. If there exists a nonempty compact subset $D$ of $A$, and $y^{\prime} \in D$, such that $\left(T x, y^{\prime}-x\right) \cap(-\operatorname{int} C) \neq \varnothing$, for all $x \in A \backslash D$, then $V_{f}(A, F) \neq \varnothing$ and $V_{f}(A, F) \subset D$.

Proof. Let $f \in C^{*} \backslash\{0\}$. Define the set-valued maps $E, G: A \rightarrow 2^{A}$ by

$$
\begin{array}{ll}
E(y)=\{x \in A: f((s, y-x)) \geq 0 \forall s \in T x\}, & y \in A, \\
G(y)=\{x \in A: f((s, y-x)) \geq 0 \forall s \in T y\}, & y \in A,
\end{array}
$$


respectively. As for each $y \in A$, we have $y \in E(y)$, then $E(y) \neq \varnothing$. The proof of the theorem is divided into four steps.

(I) $E$ is a KKM map on $A$.

Suppose to the contrary that there exists a finite subset $\left\{y_{1}, \ldots, y_{n}\right\}$ of $A$, and there exists $\bar{x} \in \operatorname{co}\left\{y_{1}, \ldots, y_{n}\right\}$ such that $\bar{x} \notin \bigcup_{i=1}^{n} E\left(y_{i}\right)$. Then $\bar{x} \in A, \bar{x}=\sum_{i=1}^{n} t_{i} y_{i}$ for some $t_{i} \geq 0$, $1 \leq i \leq n$, with $\sum_{i=1}^{n} t_{i}=1$, and $\bar{x} \notin E\left(y_{i}\right)$, for all $i=1, \ldots, n$. Then there exist $s_{i} \in T \bar{x}$ such that

$$
f\left(\left(s_{i}, y_{i}-\bar{x}\right)\right)<0 \quad \text { for each } i=1,2, \ldots, n \text {. }
$$

Since $F(x, y)$ is $C$-convex in its second variable, we have

$$
t_{1}\left(T \bar{x}, y_{1}-\bar{x}\right)+\cdots+t_{n}\left(T \bar{x}, y_{n}-\bar{x}\right) \subset(T \bar{x}, \bar{x}-\bar{x})+C=C
$$

Let $z_{i}=\left(s_{i}, y_{i}-\bar{x}\right)$, for each $i=1,2, \ldots, n$. By (4.9), we know there exists $c \in C$, such that

$$
t_{1} z_{1}+t_{2} z_{2}+\cdots+t_{n} z_{n}=c
$$

As $f \in C^{*} \backslash\{0\}$, we have

$$
f(c) \geq 0
$$

While by (4.8), we have $f\left(t_{1} z_{1}+t_{2} z_{2}+\cdots+t_{n} z_{n}\right)<0$. This is a contraction. Hence $E$ is a KKM map on $A$.

(II) $E(y) \subset G(y)$ for all $y \in A$ and $G$ is a KKM map.

By the $f$-pseudomonotonicity of $T$, for each $y \in A$, we have $E(y) \subset G(y)$. Since $E$ is a KKM map, so is $G$.

(III) $\bigcap_{y \in A} G(y) \neq \varnothing$.

Now we show that for each $y \in A, G(y)$ is closed. Let $\left\{x_{n}\right\}$ be a sequence in $G(y)$ such that $x_{n}$ converges to $x$. By the closedness of $A$, we have $x \in A$. Since $\left\{x_{n}\right\} \subset G(y)$, then for each $s \in T y$, we have

$$
f\left(\left(s, y-x_{n}\right)\right) \geq 0
$$

As $x_{n} \rightarrow x$, and the continuity of $f$, then for each $s \in T y$, we have

$$
f((s, y-x)) \geq 0
$$

Consequently, $x \in G(y)$. Hence $G(y)$ is closed. By the assumption, we have $G\left(y^{\prime}\right) \subset D$. Then $G\left(y^{\prime}\right)$ is compact since $D$ is compact. By step (II), we know $G$ is a KKM map. By Lemma 2.7, $\bigcap_{y \in A} G(y) \neq \varnothing$.

(IV) $\bigcap_{y \in A} G(y)=\bigcap_{y \in A} E(y)$.

Because $E(y) \subset G(y)$, we have $\bigcap_{y \in A} G(y) \supset \bigcap_{y \in A} E(y)$. Now let us show that $\bigcap_{y \in A} G(y) \subset \bigcap_{y \in A} E(y)$. Let $\bar{x} \in \bigcap_{y \in A} G(y)$. For each $y \in A$, and each $s \in T y$, we have

$$
f((s, y-\bar{x})) \geq 0
$$


For any $s \in T \bar{x}$ and for each fixed $y \in A$, define the set-valued mapping $J:[0,1] \rightarrow 2^{\Upsilon}$ by

$$
J(\alpha)=(T(\alpha y+(1-\alpha) \bar{x}), y-\bar{x}) \quad \alpha \in[0,1] .
$$

We pick a sequence $\left\{\alpha_{n}\right\} \subset(0,1]$ such that $\alpha_{n} \rightarrow 0$ and set $x_{n}=\alpha_{n} y+\left(1-\alpha_{n}\right) \bar{x}$. Since $A$ is a convex set, $x_{n} \in A$ for each $n$. It is clear that $x_{n} \rightarrow \bar{x}$. Let $w=(s, y-\bar{x})$. We have $w \in J(0)$. Since $T$ is $v$-hemicontinuous, $J(\alpha)$ is lower semicontinuous at 0 . By [16], there exist $w_{n} \in J\left(\alpha_{n}\right)=$ $\left(T\left(\alpha_{n} y+\left(1-\alpha_{n}\right) \bar{x}, y-\bar{x}\right)\right)$, such that $w_{n} \rightarrow w$. As $w_{n} \in J\left(\alpha_{n}\right)$, there exist $s_{n} \in T\left(x_{n}\right)$ such that $w_{n}=\left(s_{n}, y-\bar{x}\right)$. By $w_{n} \rightarrow w$, we have $\left(s_{n}, y-\bar{x}\right) \rightarrow(s, y-\bar{x})$. By (4.14), we have

$$
\alpha_{n} f\left(\left(s_{n}, y-\bar{x}\right)\right)=f\left(\left(s_{n}, \alpha_{n} y+\left(1-\alpha_{n}\right) \bar{x}-\bar{x}\right)\right) \geq 0 .
$$

Since $\alpha_{n}>0, f\left(\left(s_{n}, y-\bar{x}\right)\right) \geq 0$. Hence $f((s, y-\bar{x})) \geq 0$ since $f$ is continuous and $w_{n} \rightarrow w$. Therefore, for any $s \in T \bar{x}$ and for each $y \in A$, we have $f((s, y-\bar{x})) \geq 0$. Hence $\bar{x} \in \bigcap_{y \in A} E(y)$. Thus $\bigcap_{y \in A} E(y)=\bigcap_{y \in A} G(y) \neq \varnothing$. This means that there exists $x \in A$, for each $s \in T x$, we have $f((s, y-x)) \geq 0$, for all $y \in A$. It follows that $x \in V_{f}(A, F)$, thus $V_{f}(A, F) \neq \varnothing$. By the proof of Theorem 4.1, we know $V_{f}(A, F) \subset D$. Since $V_{f}(A, F) \subset V_{w}(A, F)$, we have $V_{w}(A, F) \neq \varnothing$. The proof of the theorem is completed.

\section{Connectedness and compactness of the solutions set}

In this section, we discuss the connectedness and the compactness of the weak efficient solutions set for set-valued vector equilibrium problems and the set-valued vector HartmanStampacchia variational inequalities in normed linear space.

Theorem 5.1. Let $A$ be a nonempty closed convex subset of $X$, let $C \subset Y$ be a closed convex pointed cone with int $C \neq \varnothing$, and let $F: A \times A \rightarrow 2^{\gamma}$ be a set-valued map. Assume that the following conditions are satisfied:

(i) for each $y \in A, F(\cdot, y)$ is lower semicontinuous on $A$;

(ii) $F(x, y)$ is $C$-concave in its first variable and $C$-convex in its second variable;

(iii) $F(x, x) \subset C$, for all $x \in A$;

(iv) $\{F(x, y): x, y \in A\}$ is a bounded subset in $Y$;

(v) there exists a nonempty compact convex subset $D$ of $A$, and $y^{\prime} \in D$, such that $F\left(x, y^{\prime}\right) \cap$ $(-\operatorname{int} C) \neq \varnothing$, for all $x \in A \backslash D$.

Then $V_{w}(A, F)$ is a nonempty connected compact set.

Proof. We define the set-valued map $H: C^{*} \backslash\{0\} \rightarrow 2^{D}$ by

$$
H(f)=V_{f}(A, F), \quad f \in C^{*} \backslash\{0\} .
$$

By Theorem 4.1, for each $f \in C^{*} \backslash\{0\}$, we have $H(f) \neq \varnothing$, hence $V_{w}(A, F) \neq \varnothing$ and $V_{w}(A, F) \subset$ $D$. It is clear that $C^{*} \backslash\{0\}$ is convex, so it is a connected set. Now we prove that, for each $f \in C^{*} \backslash\{0\}, H(f)$ is a connected set. Let $x_{1}, x_{2} \in H(f)$, we have $x_{1}, x_{2} \in D$ and

$$
f\left(F\left(x_{i}, y\right)\right) \geq 0 \quad \forall y \in A
$$


Because $F(x, y)$ is $C$-concave in its first variable, for each fixed $y \in A$, and for above $x_{1}, x_{2} \in$ $D$, and $t \in[0,1]$, we have $t x_{1}+(1-t) x_{2} \in D$ since $D$ is convex, and

$$
F\left(t x_{1}+(1-t) x_{2}, y\right) \subset t F\left(x_{1}, y\right)+(1-t) F\left(x_{2}, y\right)+C
$$

Hence for each $y \in A, z \in F\left(t x_{1}+(1-t) x_{2}, y\right)$, there exist $z_{1} \in F\left(x_{1}, y\right), z_{2} \in F\left(x_{2}, y\right)$, and $c \in C$, such that $z=t z_{1}+(1-t) z_{2}+c$. As $f \in C^{*} \backslash\{0\}$ and by (5.2), we have

$$
f(z)=t f\left(z_{1}\right)+(1-t) f\left(z_{2}\right)+f(c) \geq 0 .
$$

Thus

$$
f\left(F\left(t x_{1}+(1-t) x_{2}, y\right)\right) \geq 0 \quad \forall y \in A,
$$

that is $t x_{1}+(1-t) x_{2} \in H(f)$. So $H(f)$ is convex, therefore it is a connected set.

Now we show that $H(f)$ is upper semicontinuous on $C^{*} \backslash\{0\}$. Since $D$ is a nonempty compact set, by [16], we only need to prove that $H$ is closed. Let the sequence $\left\{\left(f_{n}, x_{n}\right)\right\} \subset$ $\operatorname{Graph}(H)$ and $\left(f_{n}, x_{n}\right) \rightarrow\left(f_{0}, x_{0}\right)$, where $\left\{f_{n}\right\}$ converge to $f_{0}$ with respect to the norm topology. As $\left(f_{n}, x_{n}\right) \in \operatorname{Graph}(H)$, we have

$$
x_{n} \in H\left(f_{n}\right)=V_{f_{n}}(A, F) \text {, }
$$

that is, $f_{n}\left(F\left(x_{n}, y\right)\right) \geq 0$, for all $y \in A$. As $x_{n} \rightarrow x_{0}$ and $D$ is compact, we have $x_{0} \in D$. Since for each $y \in A, F(\cdot, y)$ is lower semicontinuous on $A$, for each fixed $y \in A$, and each $z_{0} \in F\left(x_{0}, y\right)$, there exist $z_{n} \in F\left(x_{n}, y\right)$, such that $z_{n} \rightarrow z_{0}$. From $f_{n}\left(F\left(x_{n}, y\right)\right) \geq 0$, we have

$$
f_{n}\left(z_{n}\right) \geq 0
$$

By the continuity of $f_{0}$ and $z_{n} \rightarrow z_{0}$, we have

$$
f_{0}\left(z_{n}\right) \longrightarrow f_{0}\left(z_{0}\right)
$$

Let $Q=\{F(x, y): x, y \in A\}$. By assumption, $Q$ is a bounded set in $Y$, then there exist some $M>0$, such that for each $z \in Q$, we have $\|z\| \leq M$. For any $\varepsilon>0$, because $f_{n}-f_{0} \rightarrow 0$ with respect to norm topology, there exists $n_{0} \in N$, and when $n \geq n_{0}$, we have $\left\|f_{n}-f_{0}\right\|<\varepsilon$. Therefore, there exists $n_{0} \in N$, and when $n \geq n_{0}$, we have

$$
\left|f_{n}\left(z_{n}\right)-f_{0}\left(z_{n}\right)\right|=\left|\left(f_{n}-f_{0}\right)\left(z_{n}\right)\right| \leq\left\|f_{n}-f_{0}\right\|\left\|z_{n}\right\| \leq M \varepsilon \text {. }
$$

Hence

$$
\lim _{n \rightarrow \infty}\left(f_{n}\left(z_{n}\right)-f_{0}\left(z_{n}\right)\right)=0
$$


Consequently, by (5.8), (5.10), we have

$$
\begin{aligned}
\lim _{n \rightarrow \infty} f_{n}\left(z_{n}\right) & =\lim _{n \rightarrow \infty}\left(f_{n}\left(z_{n}\right)-f_{0}\left(z_{n}\right)+f_{0}\left(z_{n}\right)\right) \\
& =\lim _{n \rightarrow \infty}\left(f_{n}\left(z_{n}\right)-f_{0}\left(z_{n}\right)\right)+\lim _{n \rightarrow \infty}\left(f_{0}\left(z_{n}\right)\right) \\
& =f_{0}\left(z_{0}\right) .
\end{aligned}
$$

By (5.7), we have $f_{0}\left(z_{0}\right) \geq 0$. So for any $y \in A$ and for each $z_{0} \in F\left(x_{0}, y\right)$, we have $f_{0}\left(z_{0}\right) \geq 0$. Hence

$$
f_{0}\left(F\left(x_{0}, y\right)\right) \geq 0 \quad \forall y \in A
$$

This means that

$$
x_{0} \in V_{f_{0}}(A, F)=H\left(f_{0}\right) .
$$

Hence the graph of $H$ is closed. Therefore, $H$ is a closed map. By [16], $H$ is upper semicontinuous on $C^{*} \backslash\{0\}$. Because $F(x, y)$ is $C$-convex in its second variable, by Lemma 2.8, for each $x \in A, F(x, A)+C$ is convex. It follows from Theorem 3.1 that

$$
V_{w}(A, F)=\bigcup_{f \in C^{*} \backslash\{0\}} V_{f}(A, F)
$$

Thus by [17, Theorem 3.1] $V_{w}(A, F)$ is a connected set.

Now, we show that $V_{w}(A, F)$ is a compact set. We first show that $V_{w}(A, F)$ is a closed set. Let $\left\{x_{n}\right\} \subset V_{w}(A, F)$ with $x_{n} \rightarrow x_{0}$. Since $D$ is compact, $x_{0} \in D$. We claim that $x_{0} \in V_{w}(A, F)$. Suppose to the contrary that $x_{0} \notin V_{w}(A, F)$, then there exist some $y_{0} \in A$ such that

$$
F\left(x_{0}, y_{0}\right) \cap(-\operatorname{int} C) \neq \varnothing \text {. }
$$

Thus there exists $z_{0} \in F\left(x_{0}, y_{0}\right)$ such that

$$
z_{0} \in-\operatorname{int} C
$$

Hence -int $C$ is a neighborhood of $z_{0}$. Since $F\left(\cdot, y_{0}\right)$ is lower semicontinuous at $x_{0}$, there exists some neighborhood $U\left(x_{0}\right)$ of $x_{0}$ such that

$$
F\left(x, y_{0}\right) \cap(-\operatorname{int} C) \neq \varnothing \quad \forall x \in U\left(x_{0}\right) \cap A .
$$

Since $x_{n} \rightarrow x_{0}$, there exist some $n_{0}$, and when $n \geq n_{0}$, we have $x_{n} \in U\left(x_{0}\right) \cap A$. By (5.17),

$$
F\left(x_{n}, y_{0}\right) \cap(-\operatorname{int} C) \neq \varnothing .
$$


This contradicts $\left\{x_{n}\right\} \subset V_{w}(A, F)$. Thus $x_{0} \in V_{w}(A, F)$. This means that $V_{w}(A, F)$ is a closed set. Since $D$ is compact and $V_{w}(A, F) \subset D, V_{w}(A, F)$ is compact.

Theorem 5.2. Let $A$ be a nonempty closed convex subset of $X$, and let $C \subset Y$ be a closed convex pointed cone with int $C \neq \varnothing$. Assume that for each $f \in C^{*} \backslash\{0\}, T: A \rightarrow 2^{L(X, Y)}$ is a v-hemicontinuous, $f$-pseudomonotone mapping. Moreover, assume that the set-valued map $F: A \times A \rightarrow 2^{Y}$ defined by $F(x, y)=(T x, y-x)$ is C-convex in its second variable, and the set $\{F(x, y): x, y \in A\}$ is a bounded set in $Y$. If there exists a nonempty compact convex subset $D$ of $A$, and $y^{\prime} \in D$, such that $\left(T x, y^{\prime}-x\right) \cap(-\operatorname{int} C) \neq \varnothing$, for all $x \in A \backslash D$, then $V_{w}(A, F)$ is a nonempty connected set.

Proof. We define the set-valued map $H: C^{*} \backslash\{0\} \rightarrow 2^{D}$ by

$$
H(f)=V_{f}(A, F) \text { for each } f \in C^{*} \backslash\{0\} .
$$

By Theorem 4.2, for each $f \in C^{*} \backslash\{0\}$, we have $H(f)=V_{f}(A, F) \neq \varnothing$ and $V_{f}(A, F) \subset D$. Hence $V_{w}(A, F) \neq \varnothing$ and $V_{w}(A, F) \subset D$. Clearly, $C^{*} \backslash\{0\}$ is a convex set, hence it is a connected set. Define the set-valued maps $E, G: A \rightarrow 2^{A}$ by

$$
\begin{aligned}
& E(y)=\{x \in A: f((s, y-x)) \geq 0, \forall s \in T x\}, \quad y \in A, \\
& G(y)=\{x \in A: f((s, y-x)) \geq 0, \forall s \in T y\}, \quad y \in A,
\end{aligned}
$$

respectively. Now we prove that for each $f \in C^{*} \backslash\{0\}, H(f)$ is a connected set. Let $x_{1}, x_{2} \in$ $H(f)=V_{f}(A, F)$, then $x_{1}, x_{2} \in \bigcap_{y \in A} E(y)$. By the proof of Theorem 4.2, we have $\bigcap_{y \in A} G(y)=$ $\bigcap_{y \in A} E(y)$, so $x_{1}, x_{2} \in \bigcap_{y \in A} G(y)$. Hence for $i=1,2$, and for each $y \in A, s \in T y$, we have

$$
f\left(\left(s, y-x_{i}\right)\right) \geq 0
$$

Then, for each $y \in A, s \in T y$, and $t \in[0,1]$, we have $t x_{1}+(1-t) x_{2} \in D$ since $D$ is convex and

$$
f\left(\left(s, y-\left(t x_{1}+(1-t) x_{2}\right)\right)\right) \geq 0
$$

Hence $t x_{1}+(1-t) x_{2} \in \bigcap_{y \in A} G(y)=\bigcap_{y \in A} E(y)$. Thus $t x_{1}+(1-t) x_{2} \in H(f)$. Consequently, for each $f \in C^{*} \backslash\{0\}, H(f)$ is a convex set. Therefore, it is a connected set. The following is to prove that $H$ is upper semicontinuous on $C^{*} \backslash\{0\}$. Since $D$ is a nonempty compact set, by [16] we only need to show that $H$ is a closed map. Let sequence $\left\{\left(f_{n}, x_{n}\right)\right\} \subset \operatorname{Graph}(H)$ and $\left(f_{n}, x_{n}\right) \rightarrow\left(f_{0}, x_{0}\right)$, where $\left\{f_{n}\right\}$ converges to $f_{0}$ with respect to the norm topology of $Y^{*}$. As $\left(f_{n}, x_{n}\right) \in \operatorname{Graph}(H)$, we have

$$
x_{n} \in H\left(f_{n}\right)=V_{f_{n}}(A, F) .
$$

Then, for each $s^{\prime} \in T x_{n}$, we have that

$$
f_{n}\left(\left(s^{\prime}, y-x_{n}\right)\right) \geq 0 \quad \forall y \in A .
$$


By assumption, for each $n, T: A \rightarrow 2^{L(X, Y)}$ is $f_{n}$-pseudomonotone, and by (5.24), for each $y \in A$, for the above $x_{n}$, and for each $s \in T y$, we have

$$
f_{n}\left(\left(s, y-x_{n}\right)\right) \geq 0 \quad \forall y \in A .
$$

As $x_{n} \rightarrow x_{0}$, we have $\left(s, y-x_{n}\right) \rightarrow\left(s, y-x_{0}\right)$, and $f_{0}\left(\left(s, y-x_{n}\right)\right) \rightarrow f_{0}\left(\left(s, y-x_{0}\right)\right)$. As $x_{n} \rightarrow x_{0}$, and $D$ is compact, we have $x_{0} \in D$. Let $Q=\{F(x, y): x, y \in A\}$. By assumption, $Q$ is a bounded set in $Y$. Then, there exists $M>0$, such that for each $z \in Q$, we have $\|z\| \leq M$. For any $\varepsilon>0$, because $f_{n}-f_{0} \rightarrow 0$ with respect to the norm topology, there exists $n_{0} \in N$, and when $n \geq n_{0}$, we have $\left\|f_{n}-f_{0}\right\|<\varepsilon$. Therefore, there exists $n_{0} \in N$, and when $n \geq n_{0}$, we have

$$
\left|f_{n}\left(\left(s, y-x_{n}\right)\right)-f_{0}\left(\left(s, y-x_{n}\right)\right)\right|=\left|\left(f_{n}-f_{0}\right)\left(\left(s, y-x_{n}\right)\right)\right| \leq M \varepsilon
$$

Hence

$$
\lim _{n \rightarrow \infty}\left(f_{n}\left(\left(s, y-x_{n}\right)\right)-f_{0}\left(\left(s, y-x_{n}\right)\right)\right)=0 .
$$

Then

$$
\begin{aligned}
\lim _{n \rightarrow \infty} f_{n}\left(\left(s, y-x_{n}\right)\right) & =\lim _{n \rightarrow \infty}\left(f_{n}\left(\left(s, y-x_{n}\right)\right)-f_{0}\left(\left(s, y-x_{n}\right)\right)+f_{0}\left(\left(s, y-x_{n}\right)\right)\right) \\
& =f_{0}\left(\left(s, y-x_{0}\right)\right) .
\end{aligned}
$$

Then, by (5.25), (5.28), we have $f_{0}\left(\left(s, y-x_{0}\right)\right) \geq 0$. Hence for each $y \in A$, and for each $s \in T y$, we have $f_{0}\left(\left(s, y-x_{0}\right)\right) \geq 0$. Since $T$ is $f_{0}$-pseudomonotone, for each $y \in A$, and for each $s^{*} \in T x_{0}$, we have $f_{0}\left(\left(s^{*}, y-x_{0}\right)\right) \geq 0$. Hence $x_{0} \in H\left(f_{0}\right)=V_{f_{0}}(A, F)$. Therefore, the graph of $H$ is closed, and $H$ is a closed map. By [16], we know that $H$ is upper semicontinuous on $C^{*} \backslash\{0\}$. Because $F(x, y)$ is $C$-convex in its second variable, for each $x \in A, F(x, A)+C$ is convex. It follows from Theorem 3.1 that

$$
V_{w}(A, F)=\bigcup_{f \in C^{*} \backslash\{0\}} V_{f}(A, F)
$$

Then, by [17, Theorem 3.1], we know that $V_{w}(A, F)$ is a connected set. The proof of the theorem is completed.

Let $N_{b}(0)$ denote the base of neighborhoods of 0 of $L(X, Y)$. By [18], for each bounded subset $Q \subset X$, and for each neighborhood $V$ of 0 in $Y$, we have

$$
W(Q, V):=\{s \in L(X, Y): s(Q) \subset V\} \in N_{b}(0) .
$$

Lemma 5.3. Let $A$ be a nonempty convex subset of $X$, and let $T: A \rightarrow 2^{L(X, Y)}$. If $T$ is lower semicontinuous on $A$, then $T$ is $v$-hemicontinuous on $A$. 
Proof. For any fixed $x, y \in A$, we need to show that the set-valued mapping

$$
J(\alpha):=(T(\alpha y+(1-\alpha) x), y-x), \quad \alpha \in[0,1]
$$

is lower semicontinuous at 0 . For any $z \in J(0)$ and for any neighborhood $U(z)$ of $z$, there exists $s_{0} \in T x$ such that $z=\left(s_{0}, y-x\right)$, and there exists a neighborhood $V$ of 0 in $Y$, such that $U(z)=z+V$. Since $T$ is lower semicontinuous at $x$, for the neighborhood

$$
U\left(s_{0}\right):=s_{0}+W(y-x, V)=s_{0}+\{s \in L(X, Y):(s, y-x) \in V\}
$$

of $s_{0}$, there exists a neighborhood $U(x)$ of $x$ such that

$$
T x^{\prime} \cap U\left(s_{0}\right) \neq \varnothing \quad \forall x^{\prime} \in U(x) \cap A .
$$

For the above $y$, since $\alpha y+(1-\alpha) x \rightarrow x$, when $\alpha \rightarrow 0$, there exists $0<\alpha_{0}<1$, and when $0<\alpha<\alpha_{0}$, we have $\alpha y+(1-\alpha) x \in U(x)$. By (5.33), we have

$$
T(\alpha y+(1-\alpha) x) \cap U\left(s_{0}\right) \neq \varnothing \quad \forall 0<\alpha<\alpha_{0} .
$$

Thus there exist $s_{\alpha} \in T(\alpha y+(1-\alpha) x)$ such that $s_{\alpha} \in U\left(s_{0}\right)$, for all $0<\alpha<\alpha_{0}$. By (5.32), we have

$$
\left(s_{\alpha}, y-x\right) \in U(z) \quad \forall 0<\alpha<\alpha_{0}
$$

that is

$$
J(\alpha) \cap U(z) \neq \varnothing \quad \forall 0 \leq \alpha<\alpha_{0} .
$$

This means that $J$ is lower semicontinuous at 0 . By definition, $T$ is $v$-hemicontinuous on A.

Theorem 5.4. Let $A$ be a nonempty closed bounded convex subset of $X$, and let $C \subset Y$ be a closed convex pointed cone with int $C \neq \varnothing$. Assume that for each $f \in C^{*} \backslash\{0\}, T: A \rightarrow 2^{L(X, Y)}$ is a $f$ pseudomonotone, lower semicontinuous mapping. Moreover, assume that the set-valued map $F: A \times$ $A \rightarrow 2^{\gamma}$ defined by $F(x, y)=(T x, y-x)$ is $C$-convex in its second variable, and the set $\{F(x, y)$ : $x, y \in A\}$ is a bounded set in $Y$. If there exists a nonempty compact convex subset $D$ of $A$, and $y^{\prime} \in D$, such that $\left(T x, y^{\prime}-x\right) \cap(-\operatorname{int} C) \neq \varnothing$, for all $x \in A \backslash D$, then $V_{w}(A, F)$ is a nonempty connected compact set.

Proof. By Lemma 5.3 and Theorem 5.2, $V_{w}(A, F)$ is a nonempty connected set. Since $D$ is a compact set, we need only to show that $V_{w}(A, F)$ is closed. Let $\left\{x_{n}\right\} \subset V_{w}(A, F), x_{n} \rightarrow x_{0}$. It is clear that $x_{0} \in D$ and $\left\{x_{n}\right\} \subset D$. We claim that $x_{0} \in V_{w}(A, F)$. Suppose to the contrary that $x_{0} \notin V_{w}(A, F)$, then there exists $y_{0} \in A$ such that

$$
F\left(x_{0}, y_{0}\right) \cap(-\operatorname{int} C) \neq \varnothing,
$$


that is

$$
\left(T x_{0}, y_{0}-x_{0}\right) \cap(-\operatorname{int} C) \neq \varnothing .
$$

Thus there exists $s_{0} \in T x_{0}$ such that

$$
\left(s_{0}, y_{0}-x_{0}\right) \in-\operatorname{int} C
$$

Hence there exists $\varepsilon>0$ such that

$$
\left(s_{0}, y_{0}-x_{0}\right)+\varepsilon B \subset-\text { int } C,
$$

where $B$ is the unit ball of $Y$. Set

$$
W\left(y_{0}-A,\left(\frac{\varepsilon}{2}\right) B\right)=\left\{s \in L(X, Y):\left(s, y_{0}-A\right) \subset\left(\frac{\varepsilon}{2}\right) B\right\}
$$

By definition, $W\left(y_{0}-A,(\varepsilon / 2) B\right)$ is a neighborhood of zero of $L(X, Y)$, since $A$ is bounded. Since $T$ is lower semicontinuous at $x_{0}$, for the above $s_{0} \in T x_{0}$ and the above $W\left(y_{0}-A,(\varepsilon / 2) B\right)$, there exists a neighborhood $U\left(x_{0}\right)$ of $x_{0}$ such that

$$
T x \cap\left(s_{0}+W\left(y_{0}-A,\left(\frac{\varepsilon}{2}\right) B\right)\right) \neq \varnothing \quad \forall x \in U\left(x_{0}\right) \cap A .
$$

Since $x_{n} \rightarrow x_{0}$, there exists $N$ such that when $n \geq N$, we have $x_{n} \in U\left(x_{0}\right)$. Thus by (5.42) we have

$$
T x_{n} \cap\left(s_{0}+W\left(y_{0}-A,\left(\frac{\varepsilon}{2}\right) B\right)\right) \neq \varnothing .
$$

Thus there exist $s_{n} \in T x_{n} \cap\left(s_{0}+W\left(y_{0}-A,(\varepsilon / 2) B\right)\right)$, for all $n \geq N$. We have $s_{n}-s_{0} \in$ $W\left(y_{0}-A,(\varepsilon / 2) B\right)$, and hence

$$
\left(s_{n}-s_{0}, y_{0}-A\right) \subset\left(\frac{\varepsilon}{2}\right) B .
$$

Therefore,

$$
\left(s_{n}-s_{0}, y_{0}-x_{n}\right) \in\left(\frac{\varepsilon}{2}\right) B \quad \forall n \geq N
$$


Since $x_{n} \rightarrow x_{0}$ and $s_{0} \in L(X, Y)$, we have $\left(s_{0}, x_{0}-x_{n}\right) \rightarrow 0$. Hence there exists $N_{1} \geq N$, and when $n \geq N_{1}$, we have $\left(s_{0}, x_{0}-x_{n}\right) \in(\varepsilon / 2) B$. This combining (5.45) implies that

$$
\begin{aligned}
& \left(s_{n}, y_{0}-x_{n}\right)-\left(s_{0}, y_{0}-x_{0}\right) \\
& \quad=\left(s_{n}, y_{0}-x_{n}\right)-\left(s_{0}, y_{0}-x_{n}\right)+\left(s_{0}, y_{0}-x_{n}\right)-\left(s_{0}, y_{0}-x_{0}\right) \\
& \quad=\left(s_{n}-s_{0}, y_{0}-x_{n}\right)+\left(s_{0}, x_{0}-x_{n}\right) \in\left(\frac{\varepsilon}{2}\right) B+\left(\frac{\varepsilon}{2}\right) B=\varepsilon B \quad \forall n \geq N_{1} .
\end{aligned}
$$

Hence

$$
\left(s_{n}, y_{0}-x_{n}\right) \in\left(s_{0}, y_{0}-x_{0}\right)+\varepsilon B \quad \forall n \geq N_{1}
$$

By (5.40) and (5.47), we have

$$
\left(s_{n}, y_{0}-x_{n}\right) \in-\operatorname{int} C \quad \forall n \geq N_{1} .
$$

On the other hand, since $\left\{x_{n}\right\} \subset V_{w}(A, F)$, we have

$$
\left(T x_{n}, y-x_{n}\right) \cap(-\operatorname{int} C)=\varnothing \quad \forall y \in A, \forall n
$$

This contradicts (5.48), because $s_{n} \in T x_{n}$. Thus $x_{0} \in V_{w}(A, F)$. This means that $V_{w}(A, F)$ is a closed subset of $X$.

\section{Acknowledgments}

This research was partially supported by the National Natural Science Foundation of China and the Natural Science Foundation of Jiangxi Province, China.

\section{References}

[1] G. M. Lee, D. S. Kim, B. S. Lee, and N. D. Yen, "Vector variational inequality as a tool for studying vector optimization problems," Nonlinear Analysis: Theory, Methods E Applications, vol. 34, no. 5, pp. 745-765, 1998.

[2] Y. Cheng, "On the connectedness of the solution set for the weak vector variational inequality," Journal of Mathematical Analysis and Applications, vol. 260, no. 1, pp. 1-5, 2001.

[3] X.-H. Gong, "Efficiency and Henig efficiency for vector equilibrium problems," Journal of Optimization Theory and Applications, vol. 108, no. 1, pp. 139-154, 2001.

[4] X.-H. Gong, W. T. Fu, and W. Liu, "Super efficiency for a vector equilibrium in locally convex topological vector spaces," in Vector Variational Inequalities and Vector Equilibria, F. Giannessi, Ed., vol. 38 of Nonconvex Optimization and Its Applications, pp. 233-252, Kluwer Academic Publishers, Dordrecht, The Netherlands, 2000.

[5] X.-H. Gong, "Connectedness of the solution sets and scalarization for vector equilibrium problems," Journal of Optimization Theory and Applications, vol. 133, no. 2, pp. 151-161, 2007.

[6] Q. H. Ansari, W. Oettli, and D. Schläger, "A generalization of vectorial equilibria," Mathematical Methods of Operations Research, vol. 46, no. 2, pp. 147-152, 1997.

[7] Q. H. Ansari and J.-C. Yao, "An existence result for the generalized vector equilibrium problem," Applied Mathematics Letters, vol. 12, no. 8, pp. 53-56, 1999.

[8] I. V. Konnov and J. C. Yao, "Existence of solutions for generalized vector equilibrium problems," Journal of Mathematical Analysis and Applications, vol. 233, no. 1, pp. 328-335, 1999. 
[9] J.-Y. Fu, "Generalized vector quasi-equilibrium problems," Mathematical Methods of Operations Research, vol. 52, no. 1, pp. 57-64, 2000.

[10] S. H. Hou, H. Yu, and G. Y. Chen, "On vector quasi-equilibrium problems with set-valued maps," Journal of Optimization Theory and Applications, vol. 119, no. 3, pp. 485-498, 2003.

[11] N. X. Tan, "On the existence of solutions of quasivariational inclusion problems," Journal of Optimization Theory and Applications, vol. 123, no. 3, pp. 619-638, 2004.

[12] J.-W. Peng, H.-W. J. Lee, and X.-M. Yang, “On system of generalized vector quasi-equilibrium problems with set-valued maps," Journal of Global Optimization, vol. 36, no. 1, pp. 139-158, 2006.

[13] Q. H. Ansari and F. Flores-Bazán, "Recession methods for generalized vector equilibrium problems," Journal of Mathematical Analysis and Applications, vol. 321, no. 1, pp. 132-146, 2006.

[14] L.-J. Lin, Q. H. Ansari, and Y.-J. Huang, "Some existence results for solutions of generalized vector quasi-equilibrium problems," Mathematical Methods of Operations Research, vol. 65, no. 1, pp. 85-98, 2007.

[15] X.-J. Long, N.-J. Huang, and K.-L. Teo, “Existence and stability of solutions for generalized strong vector quasi-equilibrium problem," Mathematical and Computer Modelling, vol. 47, no. 3-4, pp. 445$451,2008$.

[16] J.-P. Aubin and I. Ekeland, Applied Nonlinear Analysis, Pure and Applied Mathematics, John Wiley \& Sons, New York, NY, USA, 1984.

[17] A. R. Warburton, "Quasiconcave vector maximization: connectedness of the sets of Pareto-optimal and weak Pareto-optimal alternatives," Journal of Optimization Theory and Applications, vol. 40, no. 4, pp. 537-557, 1983.

[18] A. P. Robertson and W. Robertson, Topological Vector Spaces, Cambridge Tracts in Mathematics and Mathematical Physics, no. 53, Cambridge University Press, New York, NY, USA, 1964. 